\title{
Tibial component with and without stem extension in a trabecular metal cone construct
}

\author{
Marrigje F. Meijer ${ }^{1} \cdot$ Alexander L. Boerboom ${ }^{1} \cdot$ Martin Stevens $^{1}$. \\ Inge H. F. Reininga ${ }^{2} \cdot$ Dennis W. Janssen $^{3} \cdot$ N. Verdonschot $^{4} \cdot$ Sjoerd K. Bulstra $^{1}$
}

Received: 21 February 2016 / Accepted: 5 August 2016 / Published online: 3 September 2016

(C) The Author(s) 2016. This article is published with open access at Springerlink.com

\begin{abstract}
Purpose The purpose of this study was to investigate stability and strain distribution of a tibial plateau reconstruction with a trabecular metal cone while the tibial component is implanted with and without a stem, and whether prosthetic stability was influenced by bone mineral density. Trabecular metal cones are designed to fill up major bone defects in total knee arthroplasty. Tibial components can be implanted in combination with a stem, but it is unclear whether this is necessary after reconstruction with a trabecular metal cone. Implanting a stem can give extra stability, but may have negative side effects.

Methods Tibial revision arthroplasties with trabecular metal cones were performed after reconstruction of a 2B bone defect according to the Anderson Orthopedic Research Institute classification. Components were implanted in seven pairs of cadaveric tibiae; one tibia of each pair was implanted with stem and the other without. All specimens were loaded to one bodyweight alternating between the medial and lateral tibial component. Implantbone micro-motions, bone strains, bone mineral density and correlations were measured and/or calculated.
\end{abstract}

Marrigje F. Meijer

M.F.Meijer@umcg.nl

1 Department of Orthopaedics, University Medical Center Groningen, University of Groningen, PO Box 30.001, 9700 RB Groningen, The Netherlands

2 Department of Trauma Surgery, University Medical Center Groningen, University of Groningen, Groningen, The Netherlands

3 Orthopedic Research Laboratory, Radboud University Medical Center Nijmegen, Nijmegen, The Netherlands

4 Laboratory of Biomechanical Engineering, University of Twente, Enschede, The Netherlands
Results Tibial components without a stem showed only more varus tilt [difference in median $0.14^{\circ}(P<0.05)$ ], but this was not considered clinically relevant. Strain distribution did not differ. Bone mineral density only had an effect on the anterior/posterior tilt $[\rho:-0.72(P<0.01)]$.

Conclusion Tibial components, with or without a stem, which are implanted after reconstruction of major bone defects using trabecular metal cones produce very similar biomechanical conditions in terms of stability and strain distribution. If in vivo studies confirm that a stem extension is not mandatory, orthopaedic surgeons can decide not to implant a stem.

Level of evidence II.

Keywords Total knee arthroplasty · Trabecular metal · Bone defect · Tibial component · TKA · TM · Stem extension $\cdot$ Cone

\section{Introduction}

Major bone defects are frequently seen in revision total knee arthroplasty (rTKA). Reasons for this may be design and removal of the primary prosthesis, original disease process, mechanism of failure and technical problems during the procedure. Reconstruction of the knee joint and acquiring correct prosthetic alignment during rTKA therefore constitute a challenging task.

Types $2 \mathrm{~B}$ and 3 bone defects according to the classification of the Anderson Orthopedic Research Institute (AORI) $[13-15,26]$ are commonly seen during rTKA and reconstruction of these major bone defects is usually done with metal augmentations in combination with a stem [6, $17,27]$, which is shown to provide a mechanically stable reconstruction [10, 29, 33]. However, the literature shows 
that a stem may cause increased stress at the distal part of the stem and a decrease in stress at the proximal part [9]. If enhanced stress-shielding occurs, adverse bone remodelling may follow in the long term, possibly influencing component fixation and inducing fractures [5, 22, 36]. Another disadvantage of the use of stems is elevated stress at the tip of the stem, which is associated with pain, lower post-operative clinical outcome and increased risk of periprosthetic fracture [1].

Trabecular metal (TM) cones [7, 8] are a relatively new option for reconstruction of major bone defects during TKA. TM, made from tantalum, is reported to be biocompatible, corrosion-resistant and highly porous, with an average pore diameter of approximately $400 \mu \mathrm{m}[2,23]$. Because of the porous structure ingrowth is encouraged when used uncemented, while fixation is solid when used with bone cement [4]. TM cones are available in various designs and sizes, in order to adjust to the type and size of the defect and bone. Several studies have shown good short-term functional results with evidence of osseointegration when a TM cone was used [11, 16, 20, 21, 25, 30, 35]. Tibial TM cones are designed to be impacted into the proximal tibia, to allow for osseous ingrowth and provide proximal support. After reconstruction of the proximal tibia, the tibial component is cemented in this cone and usually implanted with a stem. However, whether a stem is mandatory when a TM cone is used has not been investigated yet. A tibial TM cone is designed to enhance the carrying capacity of the metaphyseal bone of the proximal tibia, thereby rendering a situation as in a primary arthroplasty. Hence, a stem under the tibial component might not be needed to provide mechanical stability in combination with a tibial TM cone. Moreover, without use of a stem proximal stress-shielding may be reduced and it would save the costs of the stem.

The purposes of this study are thus to investigate stability and strain distribution of tibial reconstructions with a tibial component with and without a stem cemented on a TM cone. Additionally, it was investigated whether prosthetic stability was likely to be influenced by bone mineral density (BMD).

\section{Materials and methods}

A cadaveric study was conducted in which seven pairs of fresh-frozen tibial bones [four males and three females, mean age 82 years (range 70-89)] were disarticulated at the ankle and stripped of all soft tissues. The distal ends of the tibiae were potted in bone cement. Bone mineral density (BMD) of the tibiae was determined using a calibrated CT scan and in-house software (DCMTK). Two spherical volumes of interest with a diameter of $11.4 \mathrm{~mm}$ in the medial and lateral proximal tibia were selected for the measurements. The averaged BMD of these two regions was later used to assess whether there was any effect of BMD on the biomechanical output parameters.

In each tibia, bone cuts were made as if a primary knee prosthesis was to be placed. Secondly, an AORI type 2B defect [15] was created. On both the lateral and medial sides of the posterior rim of the tibia, a defect of approximately $1 \mathrm{~cm}^{3}$ was made. Furthermore, the proximal tibia was excochleated to simulate cancellous bone loss and the anteromedial rim was damaged, so finally a standard tibial component could not be fit stable on the cut surface, as demonstrated in Fig. 1a. In this way, the situation after removal of the primary prosthesis was simulated. The bone defect was first created in one cadaveric bone and served as a reference to create similar bone defects in the other cadaveric bones. For each pair of tibiae, one tibia was reconstructed using a porous tantalum metaphyseal full tibial cone [Trabecular Metal (TM), Zimmer Inc., Warsaw, IN, USA]. After this reconstruction a NexGen ${ }^{\circledR}$ tibial component was implanted with a $100-\mathrm{mm}$ press-fit stem extension (Zimmer Inc., Warsaw, IN, USA) and provided with a polyethylene insert [Legacy posterior stabilised (LPS) flex, 10-12 mm; Zimmer Inc., Warsaw, IN, USA]. The other tibia was reconstructed using the same TM cone and NexGen ${ }^{\circledR}$ tibial component and polyethylene insert, but no stem extension was used. All tibial components were cemented using bone cement (Refobacin ${ }^{\circledR}$ revision bone cement with clindamycin and gentamicin, Biomet Inc., Warsaw, IN, USA). Only the proximal part of the tibial component up to the connection of the stem was cemented. As bone ingrowth is impossible in this model and TM cones are frequently implanted using bone cement in clinical practice, the TM cones were also fixed by using bone cement in our experimental set-up (Fig. 1). Preparation of the tibiae and implantation of the components was performed by one orthopaedic surgeon (ALB). Allocation of whether the left or the right tibia was implanted with a stem was randomised by using a computer-generated list.

The next step was to test the cadaveric reconstructions for stability. RSA was used to determine migration and rotation of the components. Seven tantalum pellets ( $0.8 \mathrm{~mm}$ diameter) were glued to the tibial component and six tantalum pellets were placed into the shaft of the proximal and distal tibia in standard positions (Fig. 2). The tip of the polyethylene insert was chosen as the origin of the coordinate system relative to which rotations and translations of the component were expressed. Stereoradiograms of the medially and laterally loaded situations were made before loading and after 10,000 loading cycles. The radiograms were digitised manually and analysed using RSA software (RSA-CMS, Medis, Leiden, The Netherlands). In a previously conducted knee study, the estimated error for 

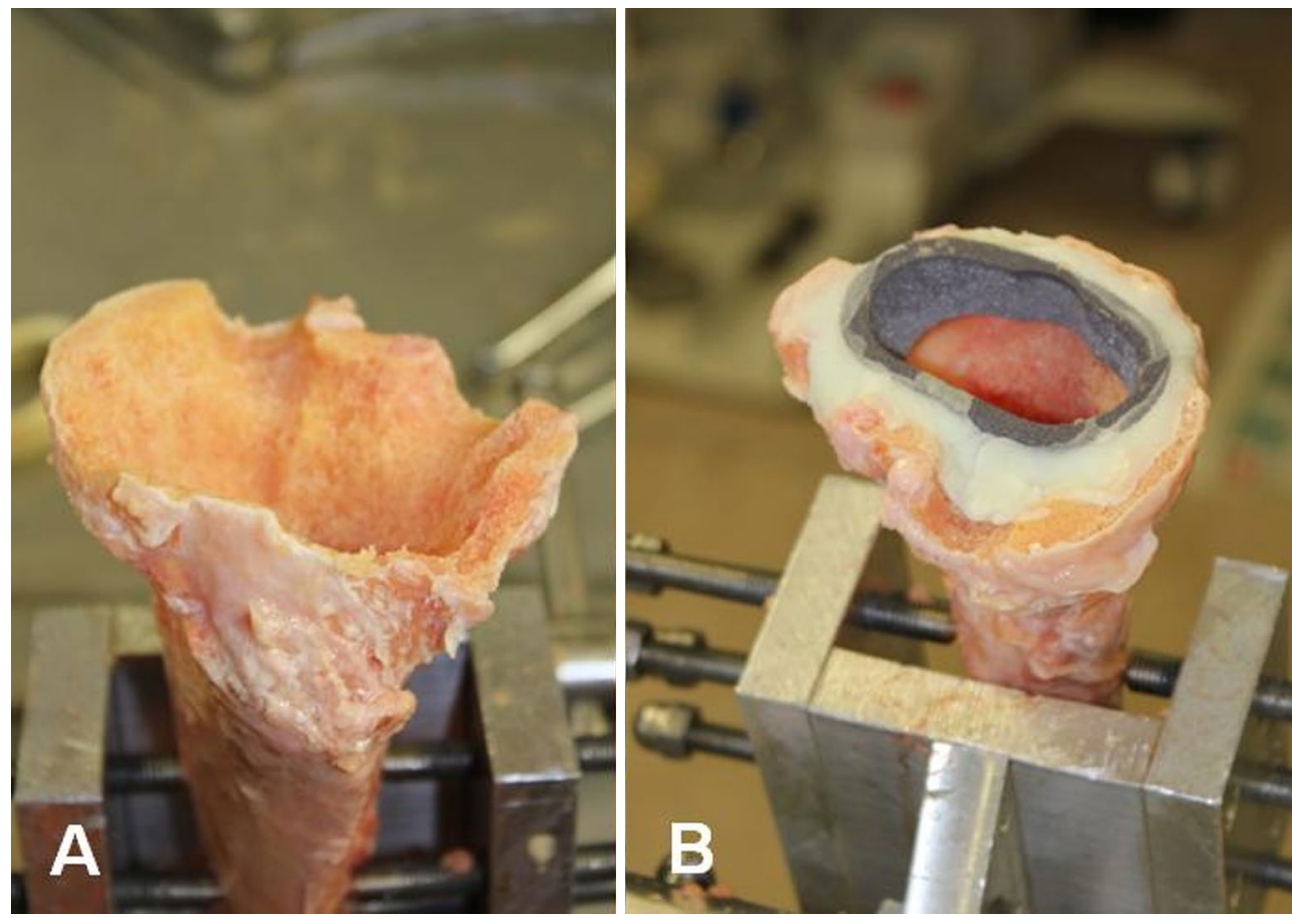

Fig. 1 a An example of the created bone defect. b Reconstruction of a bone defect using a trabecular metal cone

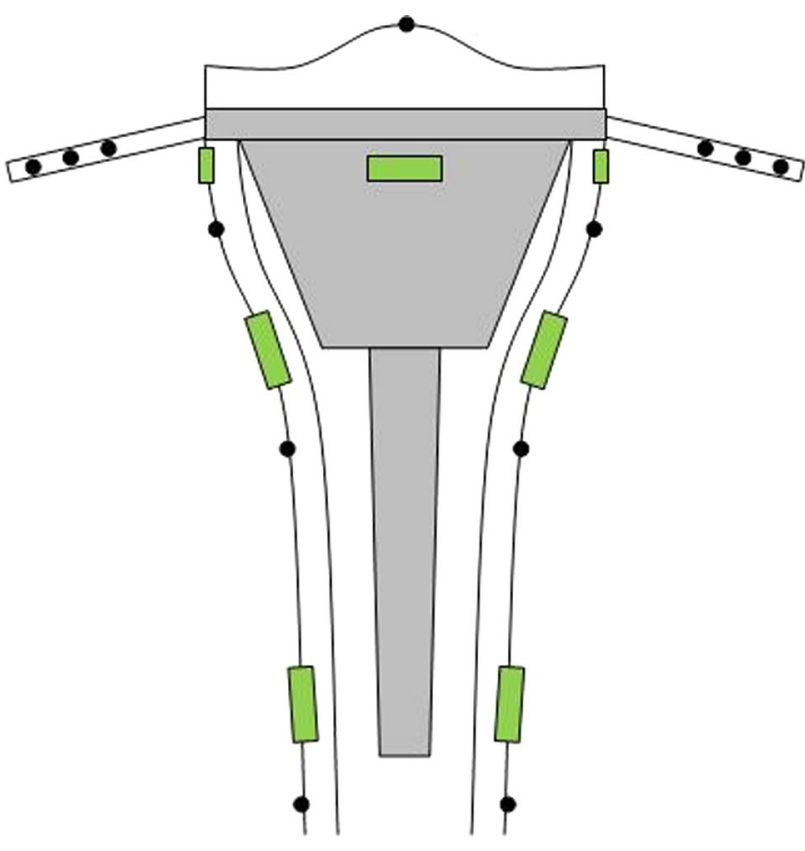

Fig. 2 Schematic representation of the locations of the tantalum pellets and strain gauges. The black dots represent the tantalum pellets and the green rectangles represent the strain gauges

the same RSA analysis was less than $50 \mu \mathrm{m}$ for repeated measurements, with a standard deviation of $0.1 \mathrm{~mm} \mathrm{[3].}$ Endpoints of the RSA were translation and rotation along the $X$-, $Y$ - and $Z$-axes. Translations along these axes were defined as medial/lateral translation, superior/inferior translation and anterior/posterior translation, respectively. Rotations along the $X-, Y$ - and $Z$-axes were defined as anterior/ posterior tilt, internal/external rotation and varus/valgus tilt, respectively. Total translation (TT) was also calculated using the following equation: $\mathrm{TT}=\sqrt{ }\left(x^{2}+y^{2}+z^{2}\right)$ [32]. The TT can be considered as a close equivalent to maximum total point motion (MTPM) [32, 34]. We calculated the TT because the MTPM was not calculated with the RSA software used in this study.

To evaluate strain distributions between the tibiae with and without stem, seven strain gauges (type YFLA-5, Tokyo Sokki Kenkyujo Co., Ltd., Tokyo) were used. The gauges were positioned horizontally on the cortex $15 \mathrm{~mm}$ under the tibial component at the medial, anterior and lateral side, and vertically at the connection of the TM cone to the stem and $5 \mathrm{~mm}$ proximally of the tip of the stem on the medial and lateral sides (Fig. 2). The contralateral tibia served as reference for the tibiae in which no stem was used. All strain gauges were connected to an amplifier and a computer to record data using monitoring software (quickDAQ 1.5.0.6, Data Translation, Inc, Marlboro, MA, USA). Strain was recorded during the entire loading session of 10,000 cycles.

The tibiae were clamped into a testing machine (MTS, model 458020, MTS Systems Corporation, Minneapolis, MN, USA) with the tibial plateau parallel to the 


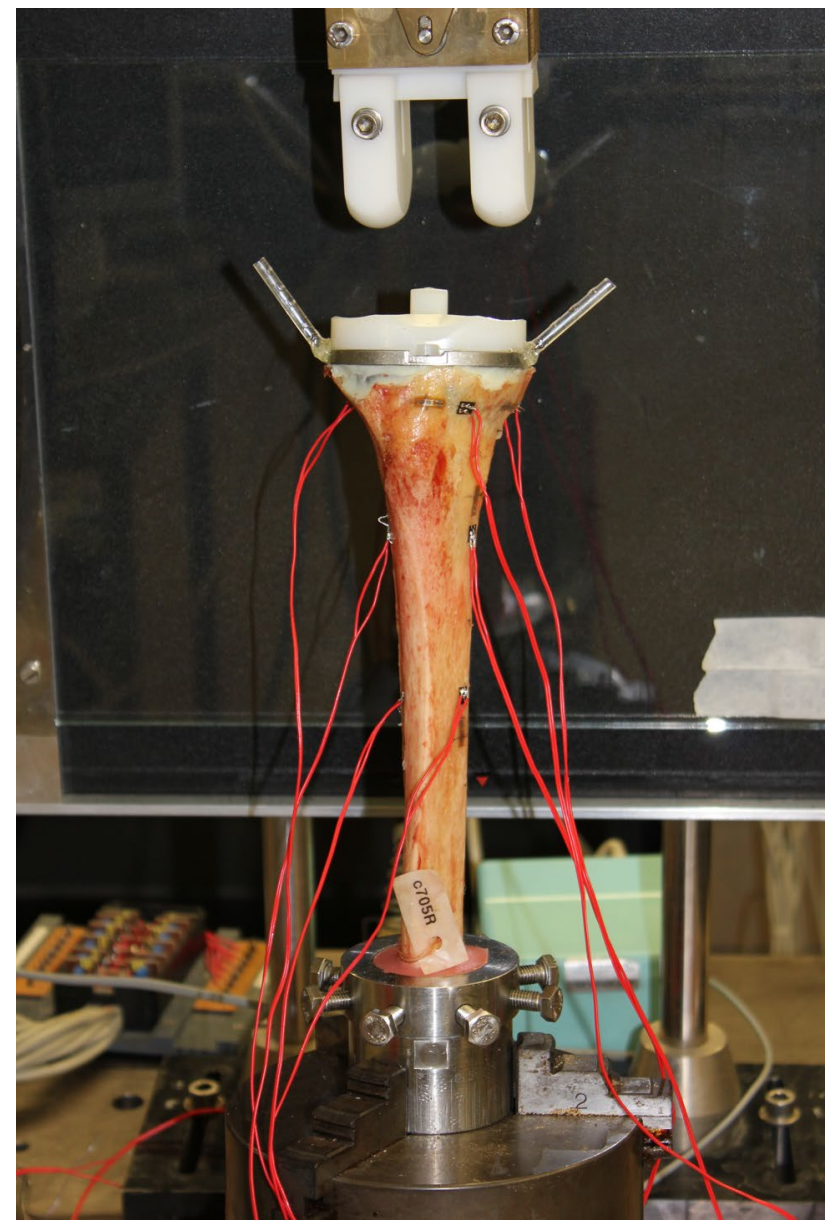

Fig. 3 Experimental set-up

working bench (Fig. 3). A unicondylar axial load alternating between the medial and lateral parts of the tibial plateau was performed. The load cycled between zero and $700 \mathrm{~N}$ at a frequency of $1 \mathrm{~Hz}$ in a series of eight loading cycles, i.e. the medial and lateral parts of the tibial plateau were both loaded eight times, thus applying varus-valgus stress to test for maximal instability of the reconstruction. A previous study at the same institution was conducted with the same set-up [24].

In accordance with regulations of the Medical Ethical Review Board of University Medical Center Groningen, ethical approval for this study was not indicated since the study was performed using cadaveric bones.

\section{Statistical analyses}

All statistical analyses were performed using the PASW software package (version 19, SPSS, Chicago). Potential differences between the two groups in rotation, translation and TT for both the medially and laterally loaded situations after 10,000 cycles were investigated using the paired Wilcoxon

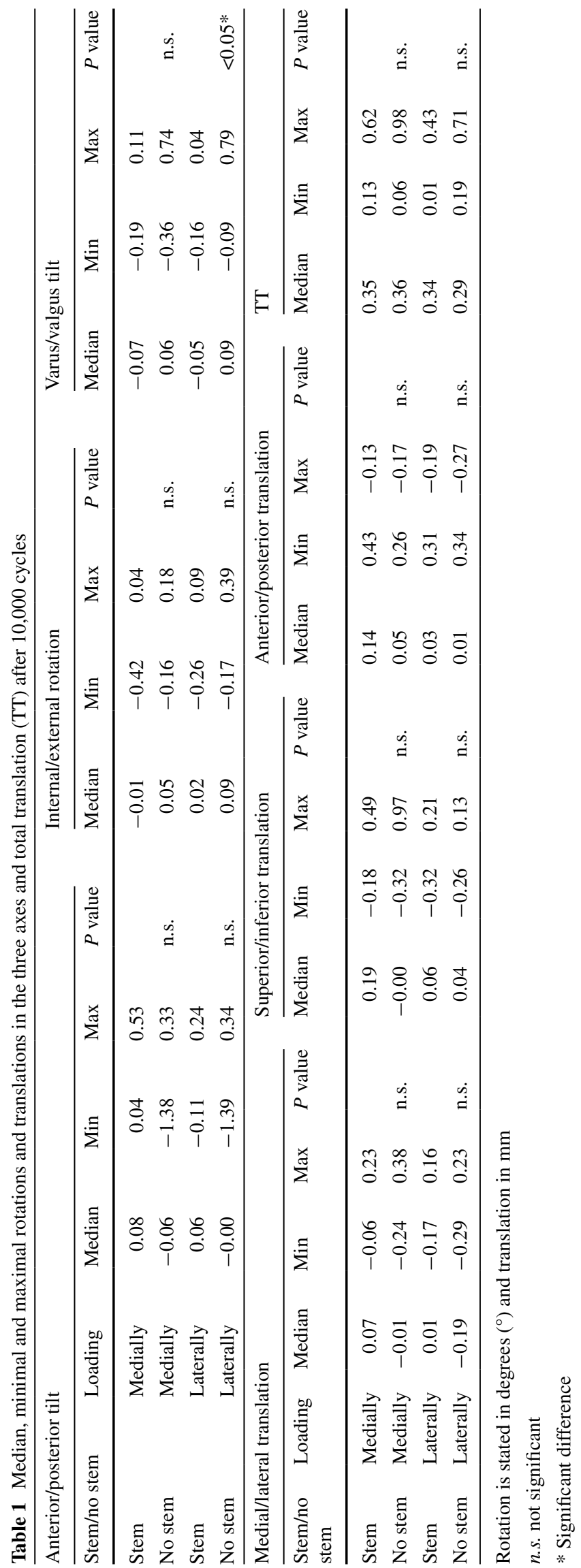


signed-rank test. The absolute differences between the medially and laterally loaded RSA measurements and the TT for these differences were also compared between the two groups using the paired Wilcoxon signed-rank test. We hypothesised that the difference in rotation or translation between the medially and laterally loaded stereoradiograms may serve as a measure of instability of the construction. The paired Wilcoxon signed-rank test was also used to compare the minimal and maximal strains at the different levels between the tibiae with and without stem. The difference between the minimal and maximal strains per level in the last 32 cycles (equal to four alternating configurations of medial and lateral load) of the loading session was also compared. Spearman's correlation coefficient $(\rho)$ was used to determine the correlation between bone mineral density and the differences in rotation, translation and TT between the medially and laterally loaded situations. The correlation coefficients were interpreted according to the benchmarks described by Domholdt [12]: $\rho 0.90-1.00$ represents a very strong correlation, $0.70-0.89$ a strong correlation, $0.50-0.69$ moderate, $0.26-0.49$ weak and $0.00-0.25$ little if any correlation [12]. A $P$ value $<0.05$ was considered to indicate statistical significance.

\section{Results}

Implanting the prosthetic material caused a fissure in one of the proximal tibiae. During loading, the proximal part of this tibia broke off. This tibia and the matching contralateral tibia were therefore excluded from further analysis. None of the other tibial components migrated visually during the loading sessions; hence, data of six pairs of tibiae were used for further analysis.
No significant differences were found for anterior/ posterior tilt or internal/external rotation between both groups after 10,000 cycles (Table 1). For varus/valgus tilt, a minimal yet significant difference was found for the laterally loaded RSA measurements. The group without a stem showed more varus tilt than the group with a stem after 10,000 cycles $(P=0.046)$. No significant difference was found for the medially loaded RSA measurements (Table 1). Translation in the three directions and TT showed no significant differences between the two groups (Table 1). When comparing the medially and laterally loaded stereoradiograms after 10,000 cycles, no significant differences in rotation or translation in any of the three directions were found (Table 2). TT did not show any significant differences between the two groups when comparing the medially and laterally loaded RSA measurements (Table 2).

A similar strain pattern was found for all strain gauges. Neither minimal strains (dip of line chart) nor maximal strains (peak of line chart) showed any significant difference between both groups for any of the locations (proximally, connection of cone to the stem or distally) (Table 3). No significant differences were found regarding the difference between the minimal and maximal strains (differences between peak and dip of line chart) between the two groups (Table 4).

Mean BMD of the cadavers was $115 \mathrm{mg} / \mathrm{mm}^{3}$ (SD 64; range 30-213). The tibia that fractured during loading had a BMD of $113 \mathrm{mg} / \mathrm{mm}^{3}$. As mentioned earlier, this tibia and the matching contralateral tibia were excluded from further analysis. The correlation between the BMD and the difference between the medially and laterally loaded RSA measurements for anterior/posterior tilt was strong $(\rho:-0.72$,
Table 2 Median, minimal and maximal differences between the medially and laterally loaded RSA measurements after 10,000 cycles for rotations and translations in the three axes and total translation (TT)

\begin{tabular}{llllll}
\hline & Stem/no stem & Median & Minimum & Maximum & $P$ value \\
\hline Anterior/posterior tilt & Stem & 0.11 & 0.01 & 0.29 & \\
Anterior/posterior tilt & No stem & 0.01 & 0.00 & 0.58 & n.s. \\
Internal/external rotation & Stem & 0.06 & 0.01 & 0.18 & \\
Internal/external rotation & No stem & 0.20 & 0.09 & 0.34 & n.s. \\
Varus/valgus tilt & Stem & 0.10 & 0.00 & 0.23 & \\
Varus/valgus tilt & No stem & 0.13 & 0.05 & 0.38 & n.s. \\
Medial/lateral translation & Stem & 0.12 & 0.04 & 0.33 & \\
Medial/lateral translation & No stem & 0.17 & 0.05 & 0.61 & n.s. \\
Superior/inferior translation & Stem & 0.25 & 0.01 & 0.58 & \\
Superior/inferior translation & No stem & 0.20 & 0.02 & 1.24 & n.s. \\
Anterior/posterior translation & Stem & 0.09 & 0.01 & 0.45 & \\
Anterior/posterior translation & No stem & 0.09 & 0.00 & 0.15 & n.s. \\
TT & Stem & 0.29 & 0.19 & 0.71 & \\
TT & No stem & 0.38 & 0.13 & 1.25 & n.s. \\
\hline
\end{tabular}

Rotation is stated in degrees $\left(^{\circ}\right)$ and translation in $\mathrm{mm}$ n.s. not significant 
Table 3 Median, minimum and maximum of the minimal and maximal strains measured during the last 32 cycles of the loading session

\begin{tabular}{|c|c|c|c|c|c|c|}
\hline Stem/no stem & Cycles & Strain & Median & Minimum & Maximum & $P$ value \\
\hline \multicolumn{7}{|c|}{ Strains proximally (medial, anterior and lateral) } \\
\hline Stem & 10,000 & Medial min & 39.57 & -97.62 & 145.11 & \\
\hline No stem & 10,000 & Medial min & 27.70 & -153.02 & 332.42 & n.s. \\
\hline Stem & 10,000 & Medial max & 76.51 & -21.11 & 179.40 & \\
\hline No stem & 10,000 & Medial max & 39.57 & -306.04 & 129.28 & n.s. \\
\hline Stem & 10,000 & Anterior min & 19.79 & -142.47 & 203.15 & \\
\hline No stem & 10,000 & Anterior min & 25.06 & -108.17 & 253.28 & n.s. \\
\hline Stem & 10,000 & Anterior max & -42.21 & -108.17 & 60.68 & \\
\hline No stem & 10,000 & Anterior max & 26.38 & -163.57 & 150.38 & n.s. \\
\hline Stem & 10,000 & Lateral min & 146.42 & -5.28 & 1155.57 & \\
\hline No stem & 10,000 & Lateral min & 26.38 & -1216.25 & 1290.12 & n.s. \\
\hline Stem & 10,000 & Lateral max & 178.08 & 65.96 & 736.08 & \\
\hline No stem & 10,000 & Lateral max & 40.89 & -817.87 & 284.93 & n.s. \\
\hline \multicolumn{7}{|c|}{ Strains at the level of the connection of cone to stem (medial and lateral) } \\
\hline Stem & 10,000 & Medial min & 48.81 & -282.30 & 356.17 & \\
\hline No stem & 10,000 & Medial min & 110.81 & -36.94 & 530.29 & n.s. \\
\hline Stem & 10,000 & Medial max & 87.06 & 60.68 & 332.42 & \\
\hline No stem & 10,000 & Medial max & 75.19 & -18.47 & 1408.84 & n.s. \\
\hline Stem & 10,000 & Lateral min & -7.92 & -124.00 & 517.10 & \\
\hline No stem & 10,000 & Lateral min & 7.92 & -226.89 & 110.81 & n.s. \\
\hline Stem & 10,000 & Lateral max & 34.30 & -81.79 & 229.53 & \\
\hline No stem & 10,000 & Lateral max & 17.15 & -197.87 & 514.46 & n.s. \\
\hline \multicolumn{7}{|c|}{ Strains distally (medial and lateral) } \\
\hline Stem & 10,000 & Medial min & 0.00 & -44.85 & 274.38 & \\
\hline No stem & 10,000 & Medial min & -1.32 & -522.38 & 44.85 & n.s. \\
\hline Stem & 10,000 & Medial max & 97.62 & 34.30 & 498.63 & \\
\hline No stem & 10,000 & Medial max & 75.19 & -2.64 & 321.87 & n.s. \\
\hline Stem & 10,000 & Lateral min & -48.81 & -279.66 & 31.66 & \\
\hline No stem & 10,000 & Lateral min & -30.34 & -139.83 & 0.00 & n.s. \\
\hline Stem & 10,000 & Lateral max & 27.70 & -29.02 & 63.32 & \\
\hline No stem & 10,000 & Lateral max & 19.79 & -34.30 & 474.89 & n.s. \\
\hline
\end{tabular}

Strain is stated in micro-strains ( $\mu$ strain)

n.s. not significant
$P<0.01$ ), indicating that for this direction more motion was produced for lower-density bones. After exclusion of one outlier, correlation was moderate and significant $(\rho$ : $-0.64, P<0.04)$, and little if any correlation existed for internal/external rotation and varus/valgus tilt $[\rho:<-0.10$, $P=$ (n.s.)]. There was little if any correlation between the BMD and the difference between the medially and laterally loaded RSA measurements for medial/lateral translation [ $\rho$ : $-0.21, P=$ (n.s.) ], a moderate and non-significant correlation for superior/inferior translation $[\rho:-0.50, P=$ (n.s.) $]$, and a weak and non-significant correlation for anterior/ posterior translation $[\rho:-0.25, P=($ n.s. $)]$. The correlation between the BMD and the TT of the difference between the medially and laterally loaded measurements was moderate and non-significant $[\rho:-0.50, P=($ n.s. $)]$.

\section{Discussion}

The most important finding of the present study was that tibial components, with or without a stem, which are implanted after reconstruction of major bone defects using TM cones produce very similar biomechanical conditions in terms of stability and strain distribution. TM cones are designed to fill up bone defects during TKA. However, it is unclear whether tibial components should be implanted with or without a stem after reconstruction of bone defects (AORI 2B/3) using a TM cone. The aim was therefore to investigate stability and strain distribution of a tibial plateau reconstruction with a TM cone while the tibial component was implanted with and without a stem. We also questioned whether prosthetic stability was influenced by BMD. 
Table 4 Median, minimal and maximal difference in strain between the minimal and maximal strains measured during the last 32 cycles of the loading session

\begin{tabular}{|c|c|c|c|c|c|}
\hline $\begin{array}{l}\text { Location of } \\
\text { strain gauge }\end{array}$ & $\begin{array}{l}\text { Stem/no } \\
\text { stem }\end{array}$ & Median & Minimum & Maximum & $P$ value \\
\hline $\begin{array}{c}\text { Proximal } \\
\text { medial }\end{array}$ & Stem & -13.19 & -176.77 & 42.21 & \\
\hline $\begin{array}{c}\text { Proximal } \\
\text { medial }\end{array}$ & No stem & 64.64 & -23.75 & 203.15 & n.s. \\
\hline $\begin{array}{r}\text { Proximal } \\
\text { anterior }\end{array}$ & Stem & 22.43 & -68.60 & 142.47 & \\
\hline $\begin{array}{r}\text { Proximal } \\
\text { anterior }\end{array}$ & No stem & 50.13 & -47.49 & 102.89 & n.s. \\
\hline $\begin{array}{c}\text { Proximal } \\
\text { lateral }\end{array}$ & Stem & 5.28 & -271.74 & 419.49 & \\
\hline $\begin{array}{c}\text { Proximal } \\
\text { lateral }\end{array}$ & No stem & -14.51 & -398.38 & 1005.18 & n.s. \\
\hline $\begin{array}{c}\text { Cone-stem } \\
\text { medial }\end{array}$ & Stem & -30.34 & -377.27 & 52.77 & \\
\hline $\begin{array}{l}\text { Cone-stem } \\
\text { medial }\end{array}$ & No stem & -9.23 & -878.55 & 68.60 & n.s. \\
\hline $\begin{array}{l}\text { Cone-stem } \\
\text { lateral }\end{array}$ & Stem & -21.11 & -182.04 & 287.57 & \\
\hline $\begin{array}{l}\text { Cone-stem } \\
\text { lateral }\end{array}$ & No stem & -27.70 & -461.70 & 2.64 & n.s. \\
\hline $\begin{array}{l}\text { Distal } \\
\text { medial }\end{array}$ & Stem & -39.57 & -532.93 & 168.85 & \\
\hline $\begin{array}{l}\text { Distal } \\
\text { medial }\end{array}$ & No stem & -52.77 & -844.25 & 13.19 & n.s. \\
\hline $\begin{array}{l}\text { Distal } \\
\text { lateral }\end{array}$ & Stem & -58.04 & -342.98 & 0.00 & \\
\hline $\begin{array}{l}\text { Distal } \\
\text { lateral }\end{array}$ & No stem & -50.13 & -614.72 & 2.64 & n.s. \\
\hline
\end{tabular}

Strain is stated in micro-strains ( $\mu$ strain)

n.s. not significant

Results of this study showed no evidence that a stem creates benefit and improves stability when a tibial component is implanted in a TM cone. Results of RSA measurements showed no difference between both groups, indicating that both constructions are stable. To our knowledge, this is the first study to investigate stability of a tibial reconstruction with a TM cone and a tibial component without stem extension. Results of RSA analysis only showed a significant difference for varus/valgus tilt of the laterally loaded RSA measurements. The cone without a stem showed more varus tilt than the cone implanted with a stem. Differences were small though. The difference between the medians of both groups was only $0.14^{\circ}$, and the range was $0.20^{\circ}$ and $0.89^{\circ}$ for tibial components with and without a stem, respectively. Since these differences were extremely small, they obviously lacked clinical significance. Rotations and translations in the other directions did not show significant differences between the two groups. We calculated differences between the medially and laterally loaded RSA measurements, as this may also give an indication of instability, but this showed no differences either.

Advantages of using stems are resistance of the tibial component to shear loads, reduced tibial lift-off and increased stability leading to reducing micro-motion. Potential disadvantages include stress-shielding with associated reduction in BMD, risk of subsidence and loosening, periprosthetic fracture and end-of-stem pain [31]. In vitro studies have demonstrated a decrease in proximal tibial strain and increase in strain at the distal tip of the stem when a stem was used $[5,9]$. In a study by Bourne et al. [5], it was concluded that tibial components should have either no short intramedullary stem or only a short one, due to negative side effects. In our study, we did not find differences in strain distribution between the two groups. Reason for this may be that we analysed bones with severe defects and reconstructed those with the cones. Apparently the addition of the stem did not add to stability or to load transfer; therefore, no strain increase at the tip of the stem or decrease at the proximal tibia was observed. This finding is consistent with our results of the RSA analysis, showing that a base plate without stem in a TM cone is a stable mechanical construction.

In this study, a low BMD strongly correlated with a larger difference between the medially and laterally loaded RSA measurements for anterior/posterior tilt. We hypothesised that a greater difference between these measurements could be an indication of instability. A low BMD may thus theoretically decrease stability of the construction. Even though the correlation between the BMD and anterior/posterior tilt was strong and significant, maximum difference in anterior/posterior tilt was only $0.58^{\circ}$. Such differences are very small and not considered clinically important. For other rotations and translations, correlations with BMD ranged from moderate to weak and were non-significant. It is therefore assumed that the results of this study are representative. Patients who undergo rTKA in general practice tend to be older, so variety in BMD can also be expected. Moreover, analyses were done in pairs (left and right tibia) per cadaver, thereby facilitating investigation of the effect of the stem despite the variability in BMD.

This study has some limitations. First of all, design was a biomechanical in vitro study using cadaveric bone. Forces and number of cycles applied are a simplification of the situation in vivo. Notwithstanding, the aim of this study was to investigate stability of the proximal tibia after bone defect repair with a tibial TM cone, for which this set-up is a suitable design. Several in vivo studies have reported good short-term functional and radiological outcome after reconstruction of bone defects using TM cones in TKA with use of stem extensions $[11,18$, 
$19,28,35]$. And yet, in vivo studies have to be conducted to gain insight into radiological and functional effects when a tibial component is implanted without a stem after reconstruction with a TM cone. Secondly, tibial TM cones in this study were implanted using bone cement. The porous structure of TM encourages bone ingrowth and can be implanted without the use of cement. From clinical experience, we have found that around $50 \%$ of the implanted tibial TM cones in our hospital use bone cement. An uncemented cone has to fit exactly when implanted; otherwise, cement has to be used. Since bone ingrowth could obviously not happen in this study and TM cones are also placed using bone cement in clinical practice, we decided to implant all TM cones using bone cement. In this way, homogeneity of the procedure is achieved and one could imagine interpreting the findings for the cementless TM cone applications as if ingrowth had occurred-as would be the case in clinical practice. Thirdly, the cadaveric bones used varied in BMD, and age of the cadavers was relatively old. This is inherent to the use of cadaveric bone, but also similar to the patient population of rTKA. BMD appeared to influence only anterior/posterior tilt. Stability in other directions was not influenced.

\section{Conclusion}

This study suggests that additional stem extension of the tibial component may not be required. In vivo studies have to be performed to gain insight into the radiological and functional effects when a tibial component is implanted without a stem after reconstruction with a TM cone. If in vivo studies confirm that a stem extension is not mandatory, orthopaedic surgeons can decide not to implant a stem.

Acknowledgments We thank Richard van Swam, Willem van de Wijdeven and Leon Driessen of the Orthopedic Research Laboratory, Radboud University Medical Center Nijmegen, for their assistance in preparing the cadavers, implanting the prosthetic material and performing the biomechanical tests. We also thank Sanaz Berahmani of the Orthopedic Research Laboratory, Radboud University Medical Center Nijmegen, for helping with the BMD measurements.

\section{Compliance with ethical standards}

Conflict of interest The Department of Orthopaedics, University of Groningen, University Medical Center Groningen, has received direct funding from the Anna Fonds (Oegstgeest, NL) for this study and Zimmer Biomet (Warsaw, IN, USA) has provided the instrumentation and tools for this study. The Department receives research institutional support from InSpine (Schiedam, NL) and Stryker (Kalamazoo, Mich. USA). One of the authors (ALB) will be and has been paid as a consultant by Zimmer Biomet (Warsaw, IN, USA) for purposes of education and training in knee arthroplasty.
Ethical approval According to the regulations of the Medical Ethical Committee of the University Medical Center Groningen ethical approval was not indicated since the study design was a cadaveric study.

Funding The Department of Orthopaedics, University of Groningen, University Medical Center Groningen, has received direct funding from the Anna Fonds (Oegstgeest, NL) for this study and Zimmer Biomet (Warsaw, IN, USA) has provided the instrumentation and tools for this study. The Department receives research institutional support from InSpine (Schiedam, NL) and Stryker (Kalamazoo, Mich. USA). One of the authors (ALB) will be and has been paid as a consultant by Zimmer Biomet (Warsaw, IN, USA) for purposes of education and training in knee arthroplasty.

Informed consent Informed consent was not applicable since the study design was a cadaveric study.

Open Access This article is distributed under the terms of the Creative Commons Attribution 4.0 International License (http://creativecommons.org/licenses/by/4.0/), which permits unrestricted use, distribution, and reproduction in any medium, provided you give appropriate credit to the original author(s) and the source, provide a link to the Creative Commons license, and indicate if changes were made.

\section{References}

1. Barrack RL, Rorabeck C, Burt M, Sawhney J (1999) Pain at the end of the stem after revision total knee arthroplasty. Clin Orthop Relat Res 367:216-225

2. Black J (1994) Biological performance of tantalum. Clin Mater 16:167-173

3. Blankevoort L, Huiskes R, de Lange A (1988) The envelope of passive knee joint motion. J Biomech 21:705-720

4. Bobyn JD, Stackpool GJ, Hacking SA, Tanzer M, Krygier JJ (1999) Characteristics of bone ingrowth and interface mechanics of a new porous tantalum biomaterial. J Bone Joint Surg Br 81:907-914

5. Bourne RB, Finlay JB (1986) The influence of tibial component intramedullary stems and implant-cortex contact on the strain distribution of the proximal tibia following total knee arthroplasty. An in vitro study. Clin Orthop Relat Res 208:95-99

6. Bush JL, Wilson JB, Vail TP (2006) Management of bone loss in revision total knee arthroplasty. Clin Orthop Relat Res 452:186-192

7. Christie MJ (2002) Clinical applications of trabecular metal. Am J Orthop (Belle Mead NJ) 31:219-220

8. Cohen R (2002) A porous tantalum trabecular metal: basic science. Am J Orthop (Belle Mead NJ) 31:216-217

9. Completo A, Fonseca F, Simoes JA (2008) Strain shielding in proximal tibia of stemmed knee prosthesis: experimental study. J Biomech 41:560-566

10. Conditt MA, Parsley BS, Alexander JW, Doherty SD, Noble PC (2004) The optimal strategy for stable tibial fixation in revision total knee arthroplasty. J Arthroplasty 19:113-118

11. Derome P, Sternheim A, Backstein D, Malo M (2014) Treatment of large bone defects with trabecular metal cones in revision total knee arthroplasty: short term clinical and radiographic outcomes. J Arthroplasty 29:122-126

12. Domholdt E (1997) Physical therapy research In: Principles and applications. WB Saunders, Philadelphia 
13. Engh GA, Parks NL (1997) The management of bone defects in revision total knee arthroplasty. Instr Course Lect 46:227-236

14. Engh GA, Ammeen DJ (1998) Classification and preoperative radiographic evaluation: knee. Orthop Clin North Am 29:205-217

15. Engh GA, Ammeen DJ (1999) Bone loss with revision total knee arthroplasty: defect classification and alternatives for reconstruction. Instr Course Lect 48:167-175

16. Howard JL, Kudera J, Lewallen DG, Hanssen AD (2011) Early results of the use of tantalum femoral cones for revision total knee arthroplasty. J Bone Joint Surg Am 93:478-484

17. Huff TW, Sculco TP (2007) Management of bone loss in revision total knee arthroplasty. J Arthroplasty 22:32-36

18. Jensen CL, Winther N, Schroder HM, Petersen MM (2014) Outcome of revision total knee arthroplasty with the use of trabecular metal cone for reconstruction of severe bone loss at the proximal tibia. Knee 21:1233-1237

19. Jensen CL, Petersen MM, Schroder HM, Flivik G, Lund B (2012) Revision total knee arthroplasty with the use of trabecular metal cones: a randomized radiostereometric analysis with 2 years of follow-up. J Arthroplasty 27:1820-1826

20. Lachiewicz PF, Bolognesi MP, Henderson RA, Soileau ES, Vail TP (2012) Can tantalum cones provide fixation in complex revision knee arthroplasty? Clin Orthop Relat Res 470:199-204

21. Long WJ, Scuderi GR (2009) Porous tantalum cones for large metaphyseal tibial defects in revision total knee arthroplasty: a minimum 2-year follow-up. J Arthroplasty 24:1086-1092

22. Lonner JH, Klotz M, Levitz C, Lotke PA (2001) Changes in bone density after cemented total knee arthroplasty: influence of stem design. J Arthroplasty 16:107-111

23. Maccauro G, Iommetti PR, Muratori F, Raffaelli L, Manicone PF, Fabbriciani C (2009) An overview about biomedical applications of micron and nano size tantalum. Recent Pat Biotechnol 3:157-165

24. Meijerink HJ, van Loon CJ, de Waal Malefijt MC, van Kampen A, Verdonschot N (2010) A sliding stem in revision total knee arthroplasty provides stability and reduces stress shielding. Acta Orthop 81:337-343

25. Meneghini RM, Lewallen DG, Hanssen AD (2008) Use of porous tantalum metaphyseal cones for severe tibial bone loss during revision total knee replacement. J Bone Joint Surg Am 90:78-84
26. Mulhall KJ, Ghomrawi HM, Engh GA, Clark CR, Lotke P, Saleh KJ (2006) Radiographic prediction of intraoperative bone loss in knee arthroplasty revision. Clin Orthop Relat Res 446:51-58

27. Qiu YY, Yan CH, Chiu KY, Ng FY (2012) Review article: treatments for bone loss in revision total knee arthroplasty. J Orthop Surg (Hong Kong) 20:78-86

28. Rao BM, Kamal TT, Vafaye J, Moss M (2013) Tantalum cones for major osteolysis in revision knee replacement. Bone Joint $\mathrm{J}$ 95:1069-1074

29. Rawlinson JJ, Closkey RF Jr, Davis N, Wright TM, Windsor R (2008) Stemmed implants improve stability in augmented constrained condylar knees. Clin Orthop Relat Res 466:2639-2643

30. Schmitz HC, Klauser W, Citak M, Al-Khateeb H, Gehrke T, Kendoff D (2013) Three-year follow up utilizing tantal cones in revision total knee arthroplasty. J Arthroplasty 28:1556-1560

31. Scott CE, Biant LC (2012) The role of the design of tibial components and stems in knee replacement. J Bone Joint Surg Br 94:1009-1015

32. Stilling M, Madsen F, Odgaard A, Romer L, Andersen NT, Rahbek O et al (2011) Superior fixation of pegged trabecular metal over screw-fixed pegged porous titanium fiber mesh: a randomized clinical RSA study on cementless tibial components. Acta Orthop 82:177-186

33. Toms AD, McClelland D, Chua L, de Waal Malefijt M, Verdonschot N, Spencer Jones R et al (2005) Mechanical testing of impaction bone grafting in the tibia: initial stability and design of the stem. J Bone Joint Surg Br 87:656-663

34. Valstar ER, Gill R, Ryd L, Flivik G, Borlin N, Karrholm J (2005) Guidelines for standardization of radiostereometry (RSA) of implants. Acta Orthop 76:563-572

35. Villanueva-Martinez M, De la Torre-Escudero B, Rojo-Manaute JM, Rios-Luna A, Chana-Rodriguez F (2013) Tantalum cones in revision total knee arthroplasty. A promising short-term result with 29 cones in 21 patients. J Arthroplasty 28:988-993

36. Willems MMM, van Lenthe GH, Verdonschot $\mathrm{N}$, de Waal Malefijt MC, van Loon CJM, Huiskens R (1999) Fixation of a stemmed femoral component of a total knee replacement strongly influences eventual bone loss. Trans ORS 29:964 